\title{
Differential Diagnostics of a Tonsillar Lymphoangiomatous Polyp
}

\author{
Stella Garvie $^{1 *}$, Omar Qassid², Georgia Karachristou ${ }^{1}$ \\ ${ }^{1}$ Kettering General Hospital, Department of Ear, Nose and Throat, Kettering, NN16 8UZ, United Kingdom. \\ ${ }^{2}$ Kettering General Hospital, Department of Cellular Pathology, Kettering, NN16 8UZ, United Kingdom.
}

\begin{abstract}
An overview of a rare clinical case of a tonsillar lymphoangiomatous polyp in a patient with a history of retinoblastoma. A sample of tonsillar tissue was taken for histological review and appropriate stainings applied to establish the aetiology of the condition and exclude certain malignancies.
\end{abstract}

Keywords: Polyp, Lymphoangiomatous polyp, Reactive polyp, Tonsillar polyp, Benign tonsillar mass, Retinoblastoma.

\section{CASE REPORT}

Our patient, an 18-year-old Caucasian lady, visited her GP with complaints of an intermittent chesty cough and sore throat for about 3-4 weeks. Upon examination, the GP noticed signs of left side tonsillitis and a large polypoid-looking mass on the right tonsil. The patient did not have any feeling of fullness or foreign body in the mouth. In addition to this, she had bilaterally mildly enlarged submandibular lymph nodes (resolved by the second appointment which was attended the next day) and no trismus or red flags presented.

As she was a known case of retinoblastoma (chemotherapy 1999; right eye enucleation) there was a certain concern regarding the risk of this mass being malignant.

The patient underwent bilateral tonsillectomy and a sample of tissue was taken for histological review.

Macroscopically, there was a $15 \mathrm{~mm}$ yellowish polypoid piece of tissue with a short stalk sitting on the dome of an enlarged tonsil measuring $28 \mathrm{~mm}$ in diameter.

Microscopically, the specimen consisted of a tonsillar tissue showing a pedunculated polyp (Fig. 1) with reactive follicular hyperplasia (Fig. 2). This comprised a bland stratified squamous epithelium lining; and lymphangiectatic channels and aggregates of small round blue cells irregularly dispersed in an oedematous fibrous stroma (Fig. 3). These blue cells expressed CD45 (LCA) (Fig. 4). The cells were a mixture of small CD20 positive B-cells, and CD3 and CD5 positive T-cells (Fig. 5 and Fig. 6). There was no evidence of association with Human Papilloma Virus (HPV) infection (Fig. 7). They were negative for synaptophysin, S100 protein and sarcoma markers (Fig. 8 and Fig. 9).

The morphology and the immunophenotype were those of a benign pedunculated tonsillar lymphangiomatous polyp.

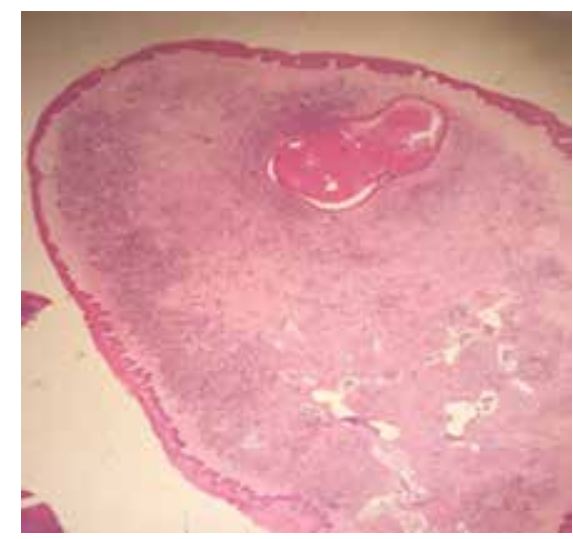

Fig. (1). The Specimen of Tonsillar Tissue With A Polyp.

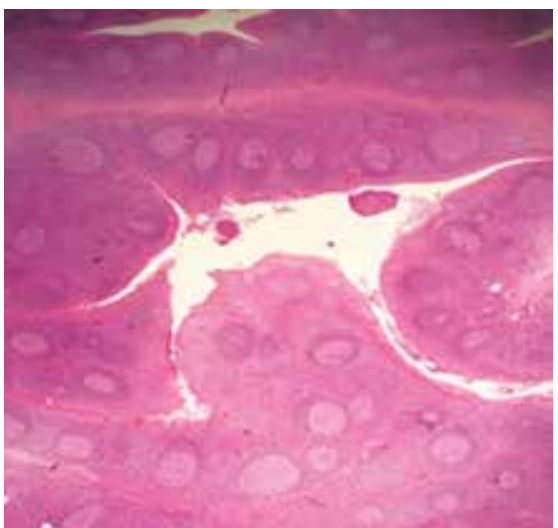

Fig. (2). Reactive Tonsillar Hyperplasia.

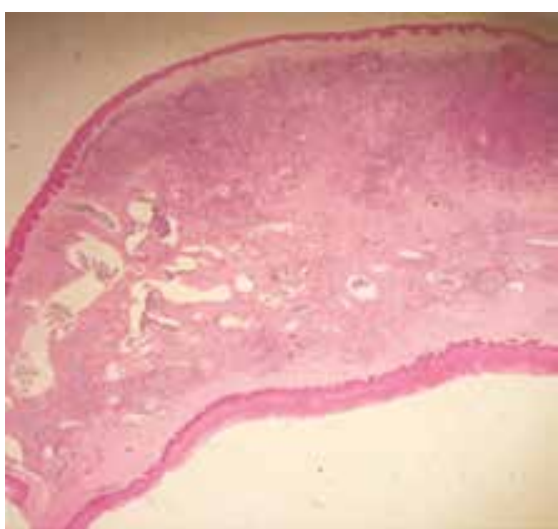

Fig. (3). Tonsillar Polyp $15 \times 10$ X $6 \mathrm{~mm}$.

\footnotetext{
*Address correspondence to this author at the Department of Ear, Nose and Throat, Kettering General Hospital, Kettering, NN16 8UZ, United Kingdom.

E-mail: stella.garvie@gmail.com
} 


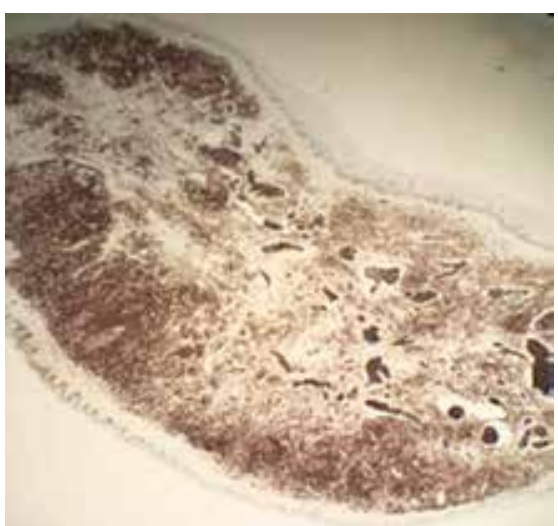

Fig. (4). LCA Positive Staining.

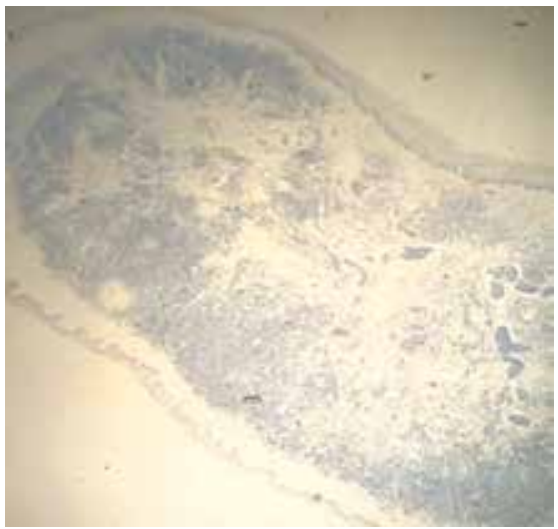

Fig. (7). HPV P-16 Negative Staining of Tonsillar Polyp Tissue.

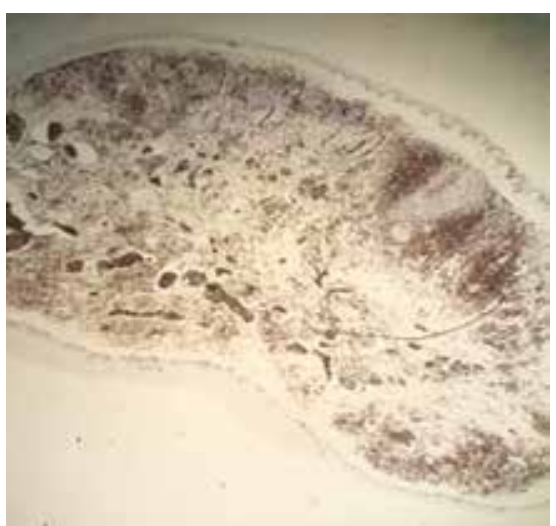

Fig. (5). CD5 Highlights Small T-cells.

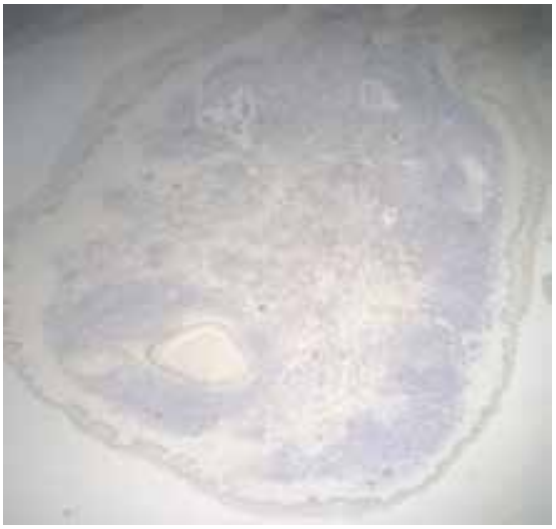

Fig. (8). Synaptophysin Negative Staining.

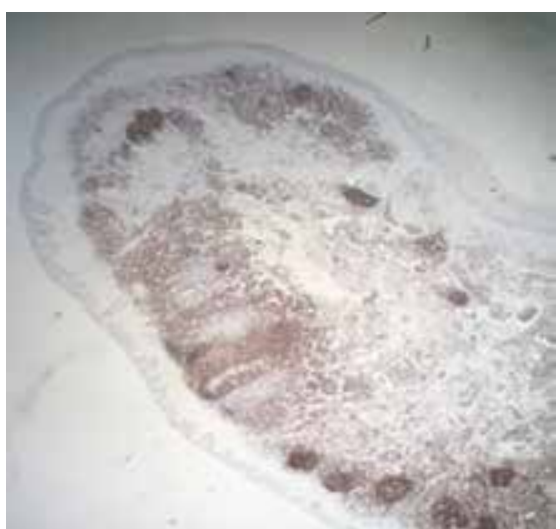

Fig. (6). CD20 Highlights Small B-cells.

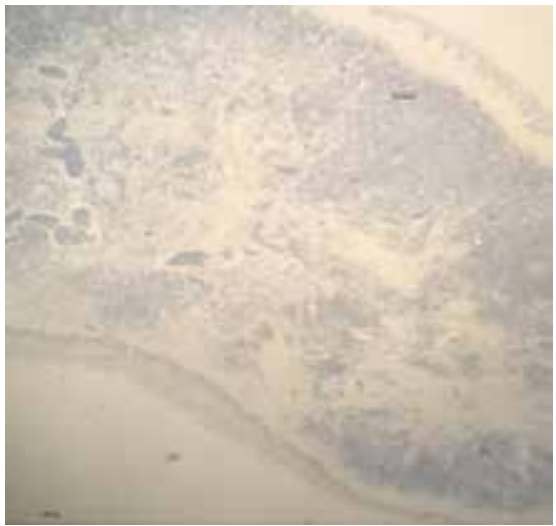

Fig. (9). S100 Protein Negative Staining.

\section{DISCUSSION}

Unlike malignant conditions of tonsils, benign tonsillar neoplasms are rare in clinical practice and often cause worry in both patients and clinicians. Specifically, pedunculated lymphoangiomatous polyp (PLP) of tonsils is extremely uncommon and there are only a few cases dedicated to this pathology $[1,2]$.

The aetiopathogenesis of PLP is not fully understood [3]; however, the clinical presentation is well described and classically involves a history of sore throat, enlarged tonsils, cervical lymphadenopathy, feeling of a foreign body or fullness in the mouth and dysphagia. Typically, routine blood tests are normal.

In our case, there were no obvious specific symptoms apart from cervical lymphoadenopathy and sore throat which were possibly related to acute pharyngitis and/or URTI rather than the presence of PLP as the duration resembled reactive lymphadenitis.

Metastatic deposits of retinoblastoma treated in the past were ruled out by using the neuroectodermal tumour marker synaptophysin (Fig. 8). It is yet unexplained if there is a connection between retinoblastoma and subsequent chemotherapy and tonsillar polyps, however, there may be a germline mutation still undiscovered.

Differentiating the tonsillar mass from retinoblastoma related oropharyngeal cancer was also important. The connection between retinoblastoma and squamous cell carcinoma can be explained by the presence of HPV. Retinoblastoma by itself is a consequence of normal cell cycle damage where mutations in the Retinoblastoma protein $(\mathrm{pRb})$ gene or MYCN oncogene play a leading role. Cell cycle proteins' expression can often be damaged by an oncovirus like HPV, a risk factor for a series of cancer but particularly for oropharyngeal cancer [4].

Although the histological picture was not suggestive of cancer, HPV testing of the specimen was performed and showed a negative result (Fig. 7). Thus, a relation between the polyp and oropharyngeal cancer was confirmed to be unlikely.

The other malignancy, which is common in young people and should be excluded when lymphoid tissue is involved in the pathological process, is lymphoma. Although the patient did not have any classical constitutional symptoms, staining for CD3, CD5, CD10, CD20 and other lymphoid markers like $\mathrm{BCL} 2$ were conducted on the biopsy material confirming the 
reactive mixture of T \& B lymphocytes (Fig. 5 and Fig. 6).

It is well known that although polyps are often associated with malignancy, initially not all polyps are neoplastic or hyperplastic in nature. They may be a consequence of the inflammatory or reactive changes in the mucosa like inflammatory polyps or a developmental change of the tissue like hamartomas which are benign and are not associated with any malignant conditions.

\section{CONCLUSION}

Pedunculated lymphoangiomatous polyp is a rare benign condition of tonsils which often causes challenges for clinicians and histologists in terms of differential diagnosis. In the presented case, it was essential to differentiate the polypoid mass from such conditions as retinoblastoma, lymphoma, oropharyngeal cancer and simply reactive changes of the tonsillar tissue. A histological examination of the lesion with a set of immunohistochemical stains was conducted on the sample to confirm the benign nature.

\section{CONFLICT OF INTEREST}

Declared none.

\section{ACKNOWLEDGEMENTS}

Declared none.

\section{REFERENCES}

[1] Khatib Y, Gite V, Patel R, et al. Lymphangiomatous polyp of palatine tonsil in a child presenting with dysphagia and dysarthria. J Clin Diagn Res 2015; 9(5): ED01-2. DOI: 10.7860/JCDR/2015/11296.5877

[2] Kardon D, Wenig B, Heffner D, et al. Tonsillar lymphangiomatous polyps: A clinicopathologic series of 26 cases. Mod Pathol 2000; 13: 1128-33. DOI: 10.1038/modpathol.3880208

[3] Ohtsuki Y, Kurita N, Iguchi M, et al. Case report: A pedunculated hamartomatous polyp of the palatine tonsil. Biomed Res 2006; 17(3): 155-58.

[4] Li W, Thompson C, O'Brien C, et al. Human papillomavirus positivity predicts favorable outcome for squamous carcinoma of the tonsils. Int J Cancer 2003; 106: 553-8. DOI: 10.1002/ijc. 11261 